\title{
Fenómenos hidrometeorológicos extremos en ciudades turísticas del norte de Quintana Roo
}

\author{
Orlando Colín Olivares* \\ Laboratorio de Observación e Investigación Espacial (UQROO) \\ Universidad Autónoma del Estado de México \\ Annie Eunice Chimal Vázquez \\ Universidad de Quintana Roo \\ Thomas Ihl \\ Universidad Halle
}

\section{Resumen}

En 1974 se creó el centro integralmente planeado de Cancún, como ciudad turística y nodo de desarrollo regional para el oriente de la Península de Yucatán, lo que generó que en los últimos 40 años se fundaran ciudades de apoyo y enclaves turísticos que se extienden desde Tulum, en el sur, hasta Puerto Juárez, en el norte, incluyendo a las islas de Cozumel y Mujeres. En esta región se concentra casi 70 \% de la población del estado y se registran los índices de crecimiento más altos del país. Estas ciudades nuevas no superan los 45 años de antigüedad, por lo que su experiencia con fenómenos naturales extremos es incipiente. Mediante un análisis geoespacial de los fenómenos que se presentan continuamente en la Península de Yucatán es posible caracterizar su tendencia de variabilidad climática y sus impactos. En ese sentido, las bases de datos y los mapas de riesgos, vulnerabilidad e impactos se vuelven herramientas imprescindibles.

\section{Palabras clave}

Caribe mexicano, ciudades, inundaciones, huracanes, turismo.

*Correo electrónico: colinolivareso@gmail.com 


\title{
External Hydrometeorology phenomena in tourism cities in Northern Quintana Roo
}

Orlando Colín Olivares Universidad Autónoma del Estado de México Annie Eunice Chimal Vázquez Universidad de Quintana Roo

Thomas Ihl Universidad Halle

\begin{abstract}
In 1974 the master-planned resort city of Cancun was created as a node for the regional development of the eastern Yucatan peninsula that resulted over the last forty years in the creation of support cities and tourism enclaves that extend form Tulum in the south to Puerto Juarez in the north, including the islands of Cozumel and Isla Mujeres. This region accounts for almost $70 \%$ of the state population and the highest growth rate in the country. These cities are less than 45 years old and their experience with extreme natural phenomena is incipient and just emerging. Through a geospatial analysis of the phenomena that happen continuously in the Yucatan peninsula, it is possible to characterize the trend of climate variability and its impacts. In this sense, database generation and hazard, vulnerability and impact maps become indispensable tools.
\end{abstract}

\section{KEY WORDS}

Floods, hurricanes, new city, Mexican Caribbean, tourism.

E-mail:colinolivareso@gmail.com 


\section{Introducción}

El cambio climático preocupa a casi todas las áreas de investigación del sector turístico. El aumento del nivel del mar, la transgresión de zonas inundadas al continente y la sustitución de la vegetación por especies de selva caducifolia en la Península de Yucatán son cambios graduales que en lo inmediato no son percibidos por la población en general, mientras que eventos extremos como sequías o huracanes se quedan en la memoria de la población (Frausto, 2014).

Mejor que reaccionar a eventos extremos es tomar medidas anticipatorias, mediante una estrategia de adaptación preventiva, flexible y participativa (Magaña y Caetano, 2007). Así, deben aprenderse lecciones de los fenómenos extremos del pasado para desarrollar estrategias de mitigación en el presente y en el futuro. Las proyecciones oficiales del cambio climático calculan un aumento gradual de la temperatura media hacia el fin del siglo entre 0.8 y $3{ }^{\circ} \mathrm{C}$, dependiendo del escenario y la localización. Los escenarios de la precipitación disminuirán hasta 30 por ciento, solo el escenario commited espera en el interior del Golfo de México un aumento de precipitación de hasta 6 por ciento. Los cambios entre los periodos son mínimos en contraste con la temperatura que registra un aumento continuo. ${ }^{1}$

Los huracanes necesitan una temperatura del agua superficial mayor de $26^{\circ} \mathrm{C}$., numerosos autores (Emanuel 2005, Webster et al. 2005, Mann y Emanuel, 2006, entre otros) han observado una relación entre la temperatura del mar, el calentamiento global y la actividad de los huracanes. De tal modo que, la diferencia entre las temperaturas del agua y del aire es decisiva.

El presente documento se enfoca en las tormentas tropicales y huracanes en cuanto fenómenos hidrometeorológicos extremos, debido a que causan grandes daños y pérdidas en el sistema de ciudades turísticas del norte de Quintana Roo. Según Konrad (2003), los huracanes provocan perjuicios por viento, objetos volantes, oleajes de tormentas y su depresión, además de erosión costera por el efecto overwash, inundaciones derivadas de las precipitaciones fuertes durante y después del impacto e incendios en la siguiente estación seca. Una consecuencia indirecta, a menudo olvidada, son los incendios, a pesar de que

${ }^{1}<$ http://zimbra.ine.gob.mx/escenarios/> [27 de noviembre de 2012]. 


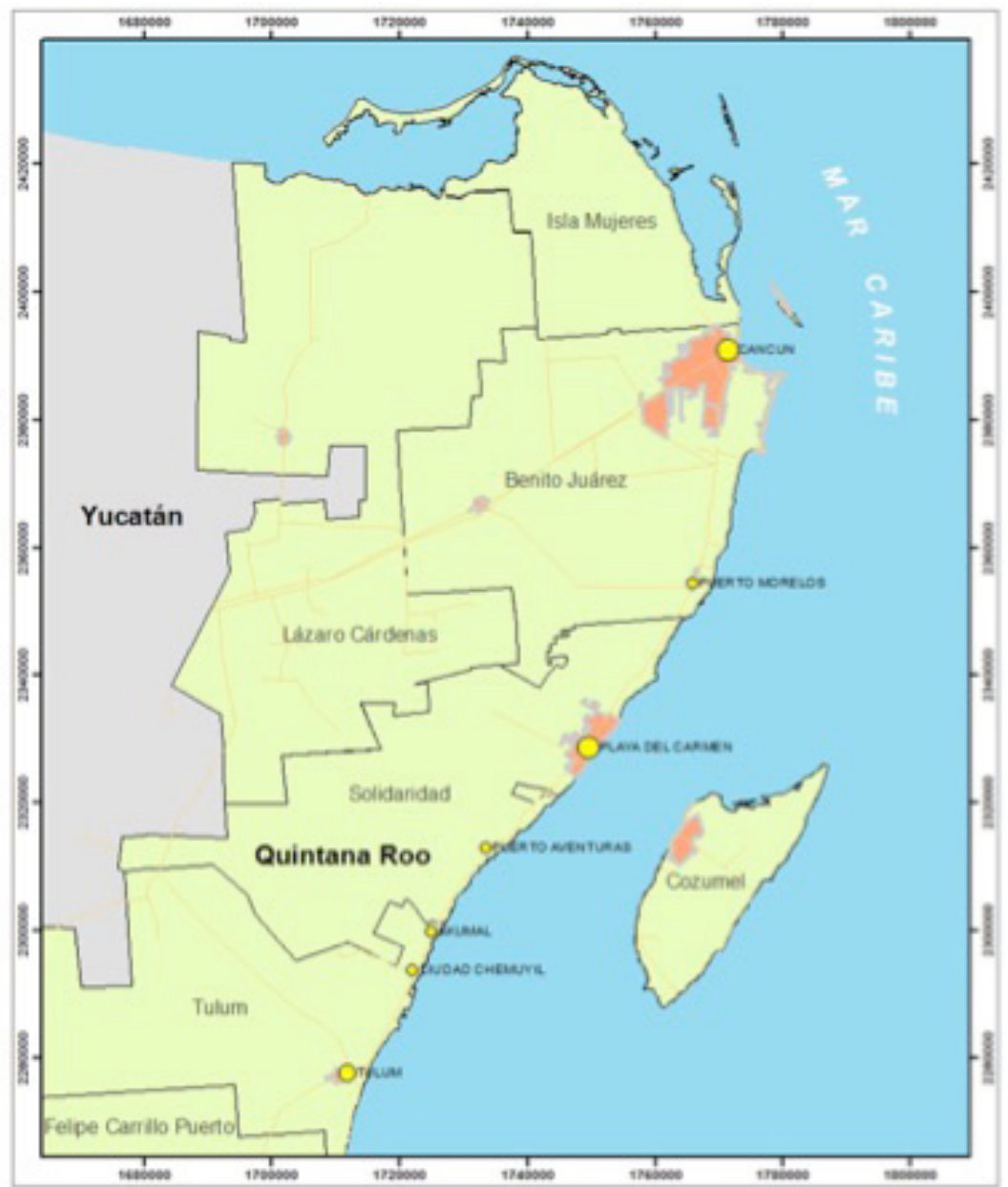

\section{LOCALIDADES TURISTICAS}
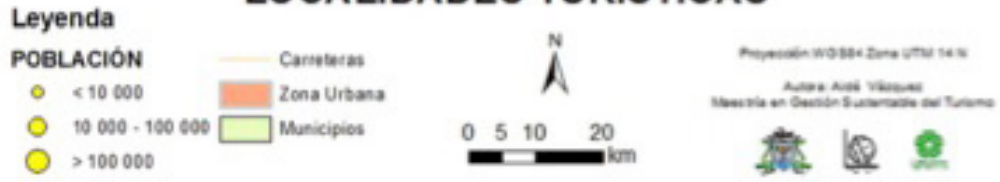

Fuente: Elaboración propia con base en datos estadísticos y vectoriales de INEGI.

Figura 1. Localidades turísticas del norte de Quintana Roo 
la temporada de sequía tras el paso de huracanes puede generar extensas áreas de madera seca vulnerable al fuego destructor (Semarnat, 2002).

\section{Problema}

El sistema de ciudades del norte de Quintana Roo comprende aquellas ubicadas entre Cancún y todas las del corredor Riviera Maya-Tulum (figura 1). Son urbes de apoyo al turismo, que concentran 70 por ciento de la población del estado (más de 850000 habitantes) y se caracterizan por ser fundadas recientemente (con excepción de Cozumel, menos de 50 años) (Rojas et al., 2012). Como parte del desarrollo de los enclaves turísticos de la región, en la última década se han observado niveles de migración del interior del país de hasta 18.5 por ciento.

Asimismo, el número y la recurrencia de huracanes y tormentas en la Península de Yucatán son progresivos y van en aumento (gráfica 1).

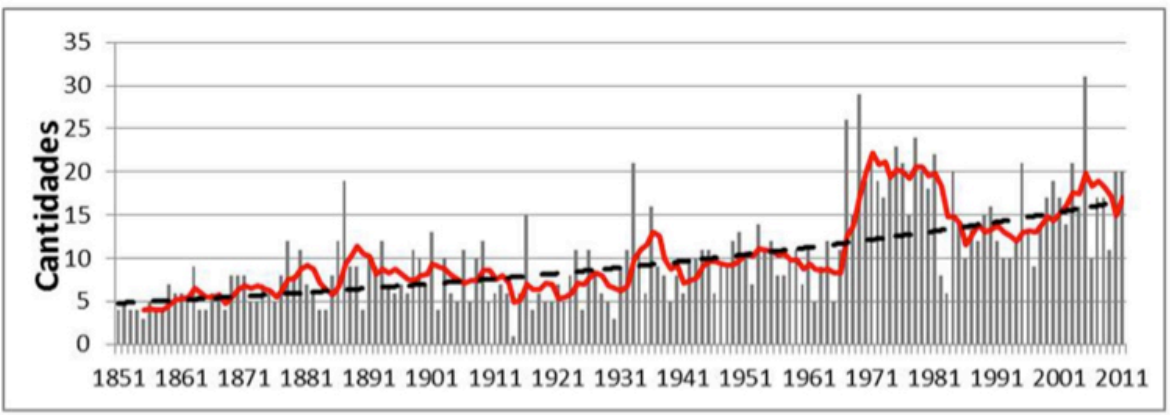

Fuente: Elaboración propia con base en NCDC-NOAA (s. f.).

Gráfica 1. Número de huracanes y tormentas al año en la cuenca del Atlántico Norte

Es necesario prevenir y planificar los riesgos y desastres en estos nuevos asentamientos ya que se carece de los instrumentos de gestión de riesgo, de autoridades formadas para la toma de decisiones y, sobre todo, de la experiencia de la población ante los peligros. 


\section{Justificación}

En los últimos años, el impacto que han provocado las tormentas y huracanes en México ha sido alarmante. La historia de estos fenómenos en la Península de Yucatán y en el estado de Quintana Roo es recurrente; solo en la última década, los huracanes Emily y Wilma afectaron en 2005 al sector de servicios de turismo y provocaron grandes pérdidas en infraestructura (Palafox y Frausto, 2008). El sistema de ciudades del norte de Quintana Roo se caracteriza por integrar localidades y municipios fundados entre 1974 y 1996, las primeras, y entre 1991 y 2008 los segundos, y esto explica la falta de experiencia en la gestión del riesgo, en especial al no contar con los instrumentos de planeación (atlas de riesgo) para la toma de decisiones en contextos de alta dinámica urbana y regional.

\section{Metodología}

Para el desarrollo de la investigación fue necesario realizar lo siguiente:

Un análisis histórico documental de las tormentas y los huracanes que han tocado tierra en la zona norte de Quintana Roo, para lo cual se recurrió a la base de datos de la Administración Nacional Oceánica y Atmosférica (National Oceanic and Atmospheric Administration, NOAA), de Estados Unidos, y se desarrolló el sistema cartográfico correspondiente.

Una revisión de las notas periodísticas locales para identificar repercusiones en los sectores social, ambiental y económico; se recabaron datos para el primer semestre de 2011, con los cuales se generaron mapas temáticos por municipio.

El desarrollo de cartografía temática base que sirviera de ejemplo para la toma de decisiones, aplicando nuevos sistemas de información e interpretación. Para la elaboración de mapas sobre impactos y fenómenos de 2011 se utilizaron los datos descritos en el punto dos, georreferenciando las variables conforme al municipio en el que ocurrieron. Se estimó la probabilidad y los efectos de huracanes y tormentas, la vulnerabilidad social y la resiliencia de las comunidades.

La identificación de prospectiva en la problemática en las nuevas ciudades turísticas del norte de Quintana Roo. 


\section{Resultados}

\section{Huracanes y tormentas en el norte de Quintana Roo}

Al analizar la trayectoria de huracanes y tormentas en la Península de Yucatán, es evidente que cada área está expuesta a peligros particulares (Ihl et al., 2008). Con el fin de obtener un mapa con amenazas específicas, es posible contar los huracanes por unidad y después clasificar el número de eventos en datos ráster. El norte de Quintana Roo muestra un peligro extremo por la acción de los huracanes (figura 2); de hecho, es la zona de mayor riesgo en la península y en México. Konrad (2003), con métodos analógicos, obtiene conclusiones similares. La mayoría de las trayectorias pasan en el estrecho marino de canal de Yucatán, entre Cabo Catoche y Cabo San Antonio, en el extremo occidente de Cuba.

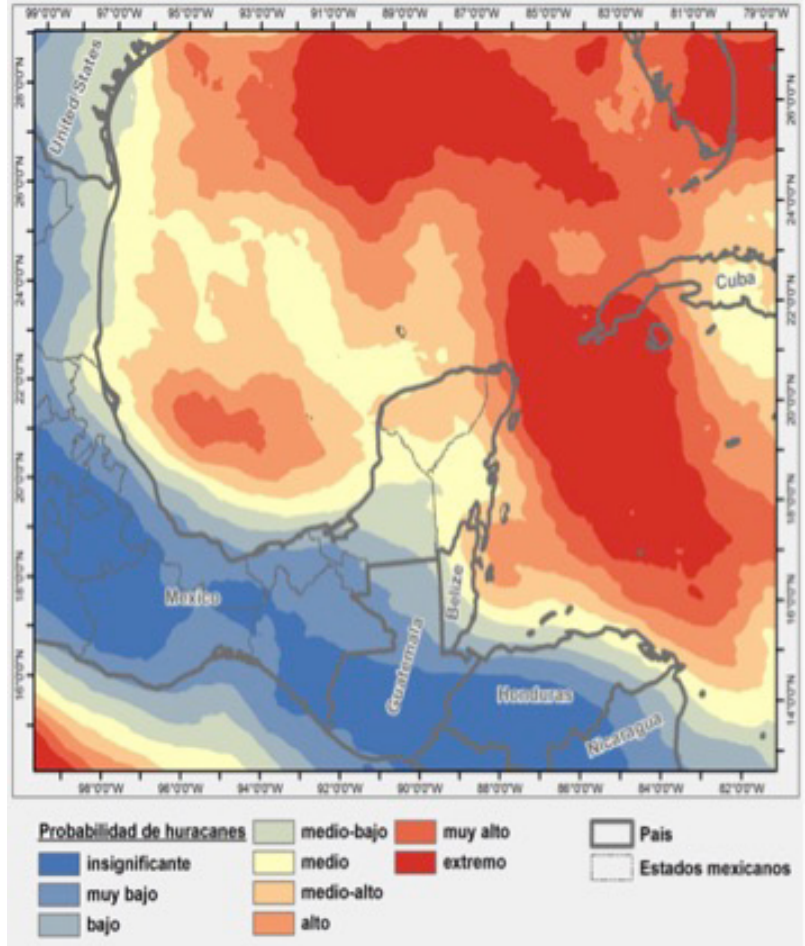

Fuente: Elaboración propia con base en NCDC-NOAA (s. f.).
Figura 2. Mapa de probabilidad del impacto de un huracán alrededor del Golfo de México 


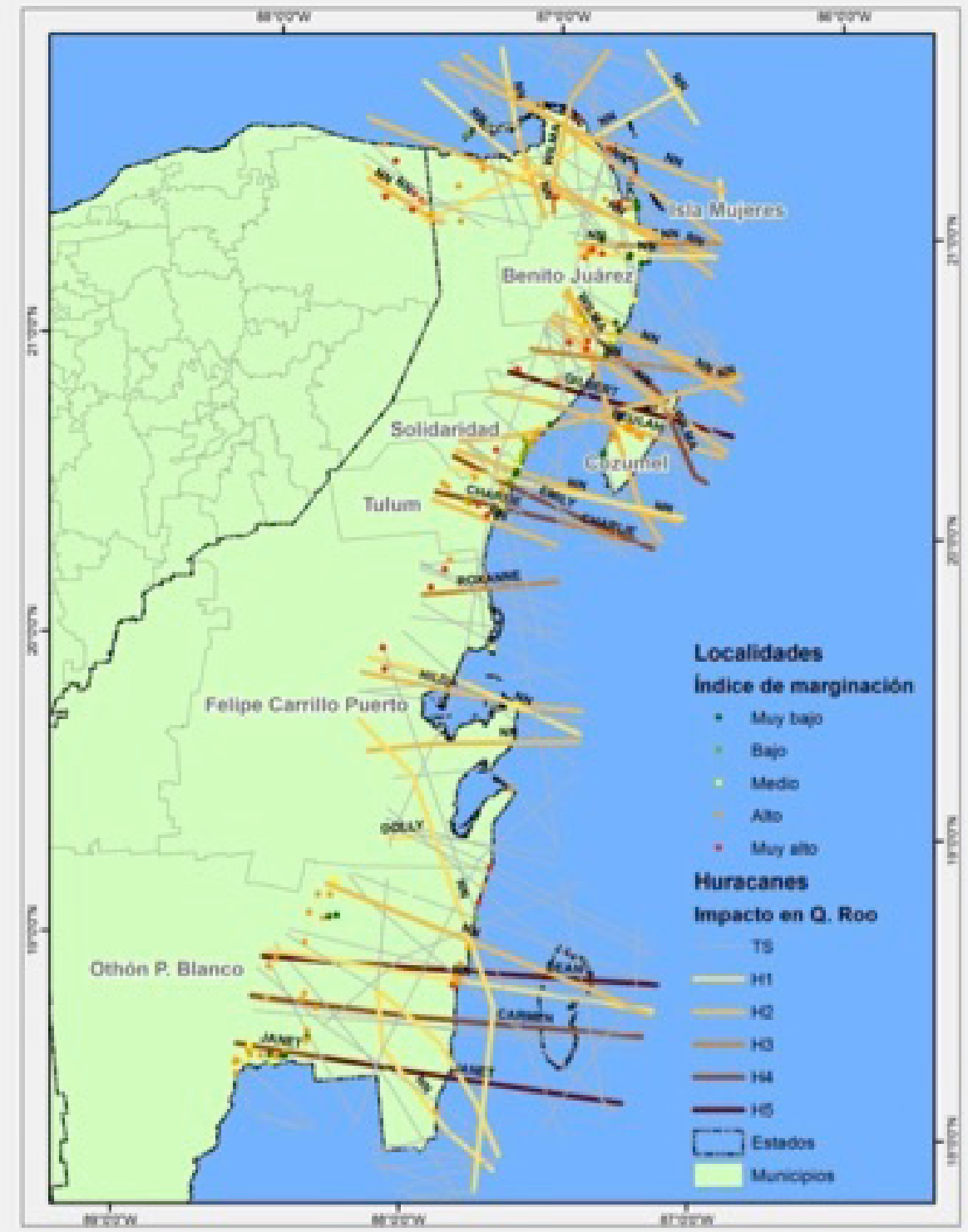

Fuente: Elaboración propia.

Figura 3. Impacto de huracanes en Quintana Roo y la resiliencia de las 
El registro de huracanes en esta región es incipiente y, por ello, para recrear los sucesos de impacto se recurre al análisis histórico de la base de datos de la NOAA. El cuadro 1 incluye la relación de huracanes de los cuales se tiene registro en la región entre 1967 y 2010.

Cuadro 1. Huracanes ordenadas por su categoría e impacto en el estado de Quintana Roo

\begin{tabular}{lccl} 
NomBRe & AÑo & Clase & Municipio \\
Gilbert & 1998 & H5 & Cozumel \\
Charlie & 1951 & H4 & Cozumel \\
Emily & 2005 & H4 & Cozumel \\
Wilma & 2005 & H4 & Cozumel, Solidaridad, B. Juárez \\
Roxanne & 1995 & H3 & F. C. Puerto, Solidaridad \\
Beulah & 1967 & H2 & Cozumel, Solidaridad \\
\hline
\end{tabular}

En la figura 3 se presentan los huracanes y las tormentas que han tocado tierra y su lugar de impacto, de acuerdo con su categoría, así como el grado de marginación de las comunidades. Destaca la afectación en la región norte del estado. Este elemento sirve para examinar la resiliencia que pueden tener estas comunidades al momento de ser aquejadas por los huracanes.

\section{Impactos generales de corto plazo}

Los medios de comunicación divulgan los daños provocados por los fenómenos hidrometeorológicos, y esto permitió reconocer, a nivel municipal, los impactos en Quintana Roo, con énfasis en el Caribe Norte. La figura 4 sintetiza los fenómenos naturales y la 5 los efectos causados por ellos. 


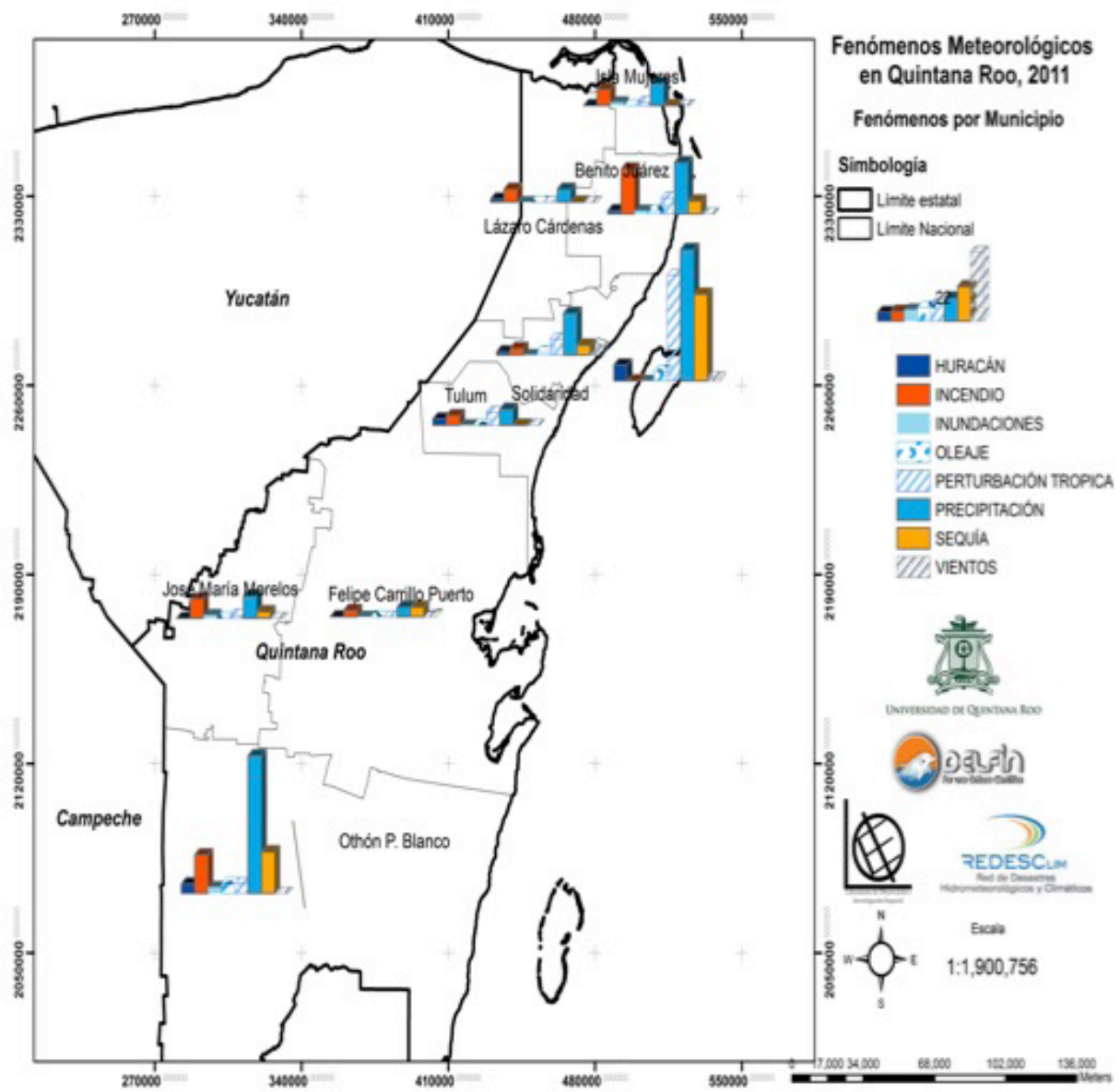

Fuente: Elaboración propia, a partir de la base de datos de fenómenos generada.

Figura 4. Fenómenos hidrometeorológicos reportados en los medios 


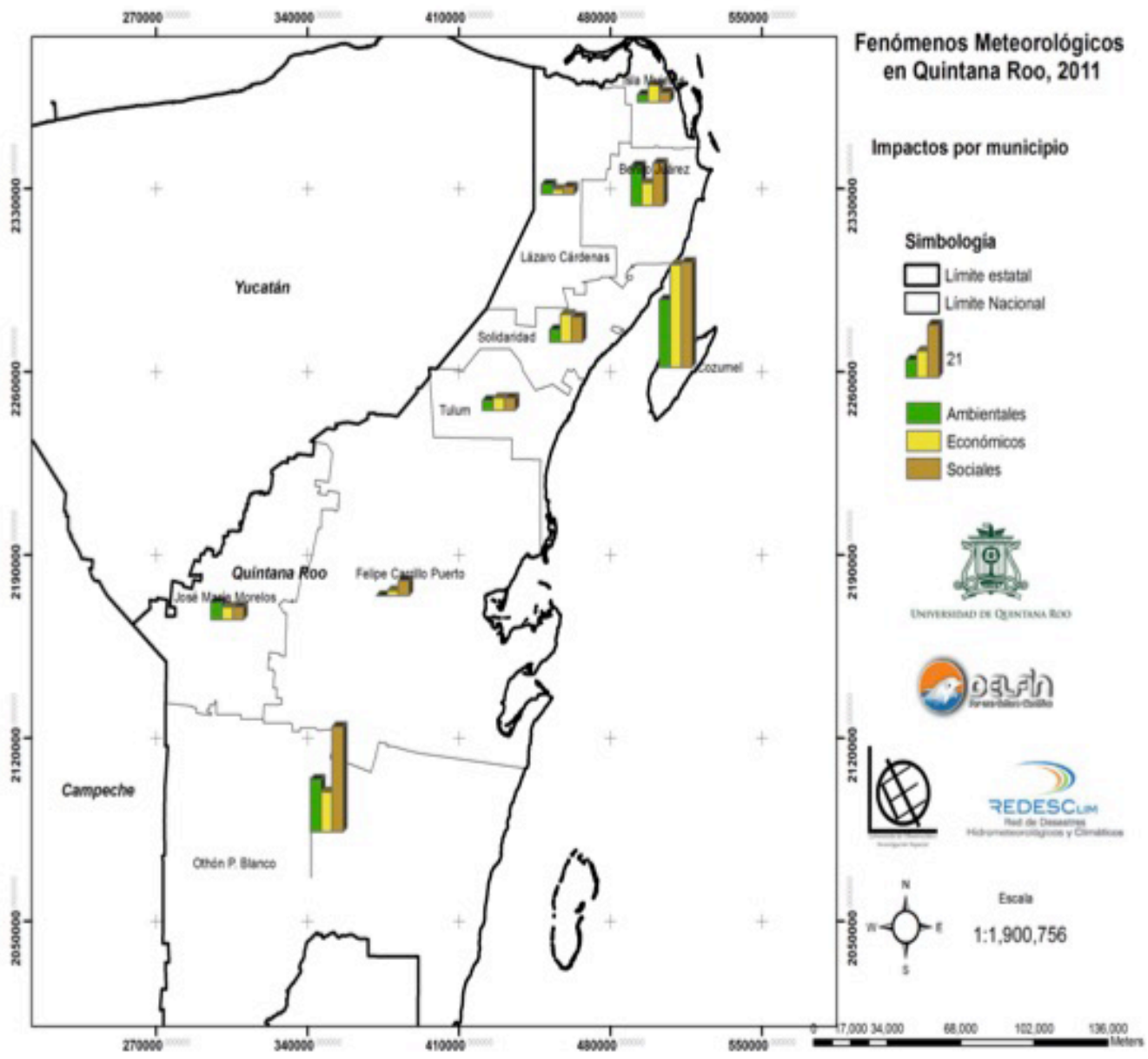

Fuente: Elaboración propia, a partir de la base de datos de fenómenos generada.

Figura 5. Impactos provocados por fenómenos hidrometeorológicos reportados por los medios

La cartografía se ha concentrado en detallar los efectos por fenómenos hidrometeorológicos en las localidades; la figura 6 muestra la vulnerabilidad al oleaje de tormentas en la zona centro de la localidad de Cozumel. 


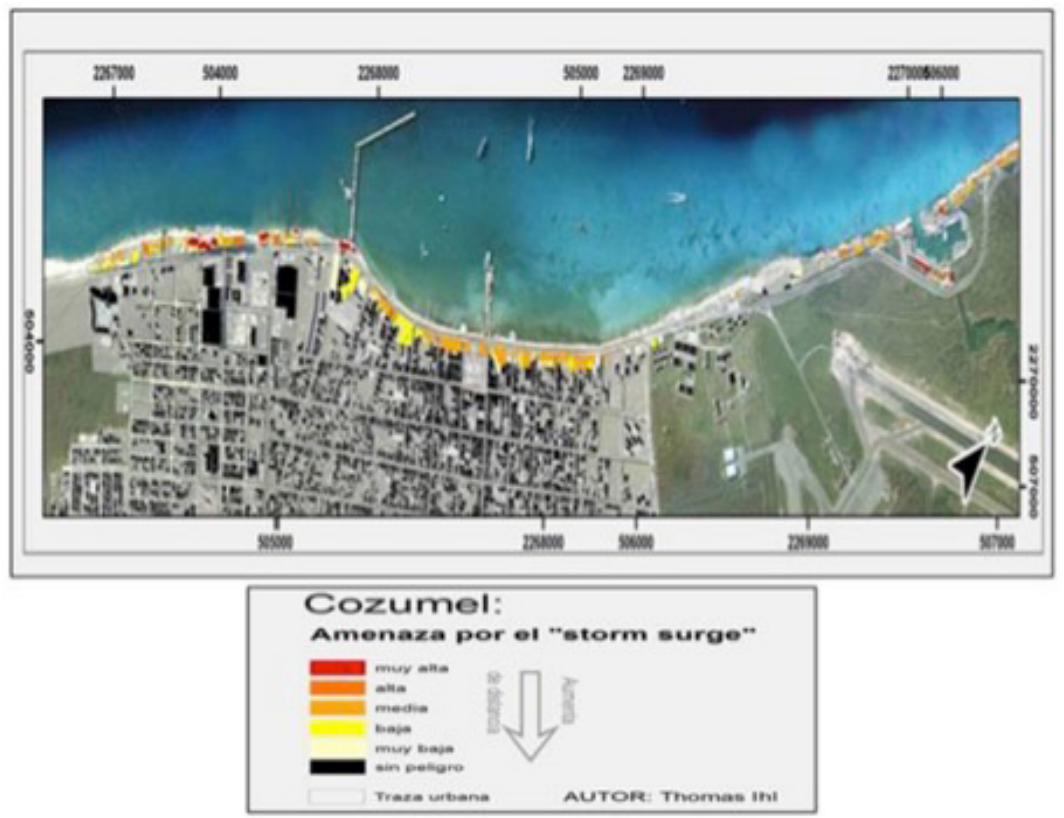

Figura 6. Amenaza por oleaje de tormenta en el malecón de Cozumel

\section{Discusión}

La generación de instrumentos para la gestión del riesgo en Quintana Roo debe ser un tema urgente de la agenda pública y científica. Hasta ahora se ha promovido la elaboración de atlas de riesgos atendiendo a parámetros de orden nacional; sin embargo, no se han considerado procesos sociales como la migración, el crecimiento exponencial, la percepción del riesgo y el conocimiento de los desastres en comunidades de nueva creación, además de que las escalas a las que se ha trabajado no permiten un análisis adecuado ni la gestión apropiada del riesgo.

Las localidades y centros urbanos del norte de Quintana Roo tienen pocos años de haber sido fundados; su experiencia en la gestión del riesgo es incipiente, como también lo son sus elementos de gestión (ayuntamientos y dependencias administrativas). En estos asentamientos urbanos, la población migrante carece del conocimiento sobre qué hacer antes, durante y después de fenómenos hidrometeorológicos extremos, lo cual aumenta el riesgo y baja su resiliencia. 
En el contexto del cambio climático, la resiliencia se denota por los grados de marginación de la población, pero también por ignorar la información sobre las zonas sujetas a riesgo y no comprender la esencia de los fenómenos naturales. Esta realidad se evidencia al desconocer el territorio en su totalidad.

\section{Conclusiones}

Las ciudades del Caribe Norte de México se han caracterizado por su crecimiento exponencial, en un entorno de migración regional y con un impulso directo de la actividad turística, lo cual refleja una disparidad entre el crecimiento económico y la planeación de las urbes.

En los discursos del cambio climático, los elementos de planificación para reducir los desastres se centran en aumentar la resiliencia de las comunidades ante estos fenómenos. Al analizar los escenarios del cambio climático, la tendencia de los huracanes y tormentas y las dinámicas de apropiación territorial, identificamos la ausencia de conocimiento de instrumentos de gestión (indicadores de riesgo) y cartográficos que apoyen la toma de decisiones.

En el contexto del cambio climático, las ciudades turísticas son esenciales para generar investigación que apoye el turismo, debido a que el ascenso del nivel del mar, la frecuencia de tormentas y sequías, la erosión de las playas y la destrucción de las costas tienen implicaciones directas en los sectores económico y ambiental, y no sólo consecuencias físicas en las zonas costeras, sino también sociales, por la pérdida de empleo, destrucción de infraestructura básica y aumento de la pobreza.

\section{Agradecimientos}

El presente escrito forma parte del proyecto "Agua y fenómenos hidrometeorológicos extremos", financiado por las REDESClim y Retac del Consejo Nacional de Ciencia y Tecnología; del programa Delfín y del cuerpo académico en Manejo de los Recursos Naturales Acuáticos del Programa de Mejoramiento del Profesorado. 


\section{Fuentes consultadas}

Emanuel, K.A. (2005). "Increasing Destructiveness of Tropical Cyclones over the Past 30 Years". Nature, 436, agosto, 686-688.

Frausto, O. (2014). Bases de datos georreferenciadas de fenómenos naturales extremos. Chetumal: Universidad de Quintana Roo (uQRoo)/Red de Desastres Hidrometeorológicos y Climáticos-Consejo Nacional de Ciencia y Tecnología (REDESClim-Conacyt). 120 pp.

Ihl, T. et al. (2008). "Identification of Geodisasters in the State of Yucatan, Mexico”. Neues Jahrbuch für Geologie und Paläontologie - Abhandlungen Band, 246 (3), febrero, 299-311.

Konrad, H.W. (2003). "Caribbean Tropical Storms. Ecological Implications for Pre-Hispanic and Contemporary Maya Subsistence on the Yucatan Peninsula”. Revista de la Universidad Autónoma de Yucatán, 18 (224/2), Impacto del Huracán Isidoro en Yucatán. Huracán, ecología y sistemas productivos (ed. especial), enero-marzo, 99-126.

Magaña, V. y E. Caetano (2007). "Pronóstico climático estacional regionalizado para la República Mexicana como elemento para la reducción de riesgo, para la identificación de opciones de adaptación al cambio climático y para la alimentación del sistema: cambio climático por estado y por sector. Informe final de trabajo” [en línea]. México: Dirección General de Investigación sobre Cambio Climático. Disponible en: http://www. ine.gob.mx/descargas/cclimatico/e2007o.pdf [2012, 21 de noviembre].

Mann, M. y K. Emanuel (2006). "Atlantic Hurricane Trends Linked to Climate Change". Eos, 87, 233-241.

NCDC-NOAA (s.f.). "International Best Track Archive for Climate Stewardship (IBTrACS)” [en línea]. National Climatic Data Center-National Oceanic and Atmospheric Administration. Disponible en: http://www.ncdc. noaa.gov/oa/ibtracs [2012, 24 de octubre].

Palafox, A. y O. Frausto (coords.) (2008). Turismo: desastres naturales, sociedad y medio ambiente. México: Secretaría de Desarrollo Social / UQRoo / Plaza y Valdés. 
Rojas, J. et al. (2012). "Dinámica demográfica de Quintana Roo y sus localidades: el surgimiento de sus ciudades intermedias y pequeñas" [en línea]. Ponencia presentada al Congreso de la Sociedad Mexicana de Demografía. Disponible en: http://www.somede.org/xireunion/ponencias/Demografia \% 20regional/79Din \% C3\% A1 mica \% 20 demogr \% C3 \% A1fica \% 20de\%20Quintana \% 20Roo \% 20y \% 20sus \% 20 localidades.pdf [2012, 20 de mayo].

Semarnat (2002). “Los huracanes y los incendios forestales” [en línea]. México: Secretaría de Medio Ambiente y Recursos Naturales. Disponible en: http://www.semarnat.gob.mx/qroo/incendios2/presentacion.shtml [2006, 9 de junio].

Webster, P.J. et al. (2005). "Changes in Tropical Cyclone Number, Duration, and Intensity in a Warming Environment”. Science, 309 (5742), septiembre, 1844-1846. 
